\title{
Software Based Implementation Methodologies for Deep Packet Inspection
}

\author{
Ajay Chaudhary ${ }^{1} \&$ Anjali Sardana ${ }^{2}$ \\ Department of Electronics \& Computer Engineering \\ Indian Institute of Technology Roorkee \\ Roorkee, India \\ ${ }^{1}$ ajaychaudhary@ieee.org, ${ }^{2}$ anjlsfec@iitr.ernet.in
}

\begin{abstract}
Deep Packet Inspection plays an important role for providing secure and congestion free network. It determines whether incoming traffic matches a database of signatures up to payload level, where each signature represents an attack, vulnerability, Virus, worm and even type of traffic. Other techniques like IDS/IPS etc uses only packet header information for decision making, while DPI is considers whole packet including payload for matching which provides better surveillance then other techniques. The problems that DPI system faces is low packet throughput, high memory requirement, latency and low accuracy at line speed of 10GbE/OC192.This paper reviews different software based approaches for efficient implementation of Deep Packet Inspection. A comparative study has been performed for these approaches on the basis of packet throughput and memory requirements. This paper is an attempt to exhaustively review existing techniques to addresses the probable research gaps. It then suggests a novel software based implementation of DPI to overcome the identified research gaps.
\end{abstract}

Keywords - deep packet Inspection; network security; string matching algorithm; survey;application layer security; deep packet inspection techniques.

\section{INTRODUCTION}

In this growing cyber world, the current state of practice regarding the technical ability to defend and monitor Internet-based attacks is not sufficient. Sophisticated attacks are almost impossible to defend using current practices. As stated by Bendrath [1] The anonymity enjoyed by today's cyber attackers poses a great threat to the global information society, the progress of information based international economy, and the advancement of global collaboration and cooperation in all areas of human endeavor. Hence accurate traffic classification and identification is key task for the network administrator to detect forbidden applications, intrusion and malicious attacks, to maintain safe network and bill on the content of traffic (for P2P or online gaming or on demand movies or music). This situation demands more than what is one by IDS/IPS and the current stateful firewall. To cope up with such situations, a more reliable and advance system is needed which can detect and stop the attack, virus and worms in the network before they harm the actual target host. To meet these demands of security and QoS techniques like DPI (Deep Packet
Inspection) help ISPs (Internet Service Providers) to serve better.

In this paper, we survey the software based Deep Packet Inspection Methodologies which includes string matching algorithm, Regular expression, finite state machine (DFA, NFA, and their improved version) then we propose a model for DPI which provide high throughput, low latency and high accuracy. The rest of the paper is organized as follows: Section 2 explains different types of packet inspection. In section 3, we address some of the key challenges for DPI. Section 4 presents existing methodologies. Section 5 describes various research gaps and in section 6, we proposed DPI implementation using soft computing technique. Paper concludes in section 7.

\section{TYPES OF PACKET INSPECTION}

\section{A. Stateful/Shallow Packet Inspection}

It is basically, a process in which the headers are parsed, and the results are compared to a rule set defined by the system administrator. It has access to Layers 3 and 4 of the OSI stack (sometimes Layer 2, as well). SPI firewalls perform the 'classic 5-tuple lookup' that is, they scan 5 tuples [2] which are source transport layer address (typically TCP or UDP), destination transport layer address (typically TCP or UDP), source IP address, destination IP address and Service type (e.g. FTP, HTTP, SMTP, POP3) These rule sets are commonly based upon above fields or certain combinations of the two and defines the type of traffic is subsequently allowed or denied.

\section{B. Medium Depth Packet Inspection}

Medium Depth Packet Inspection is done by Application proxies (AP) or gateway. An APs is located between the inside and the outside of corporate or an institution network so when any application on host inside network tries to connect with outer work its request is process through the proxy server and there is two active connection i.e. between host and AP or proxy and between proxy and real server. So each packet of data is routed through the AP. There is possibility to do limited packet filtering at Application proxies or Gateways as it typically possess the ability to do a limited amount of 
packet filtering based upon rudimentary applicationlevel data parsing.

\section{Deep Packet Inspection}

Deep Packet Inspection (DPI) is a computer network surveillance technique that uses device and technologies that inspect and take action based on the contents of the packet i.e. it consider the complete payload of packet rather than just the packet header which includes data up to layer 7 of OSI model(See Fig. 1).

The common packet inspection analyzes only the content below the layer 4 of the IP packet, including the source address, destination address, source port, destination port and the protocol type. It identifies application types in the network through the port number.

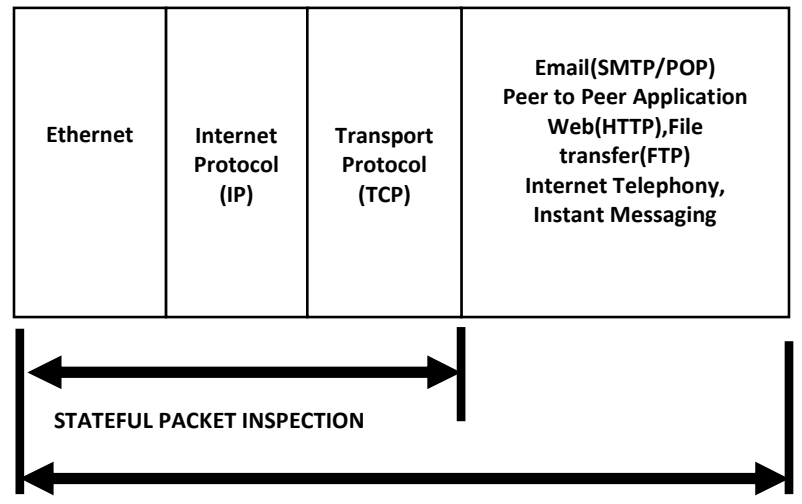

DEEP PACKET INSPECTION

Figure 1: Stateful packet inspection versus Deep packet Inspection

\section{III.SCOPE \& CHALLENGES FOR DPI}

There are several issues related to the design and implementation of deep packet inspection, basically related to the type of implementations and other challenges pertaining to performance issues. The key challenges are as follows:

\section{A. Type of DPI Implementation}

The DPI is broadly classify as[3]:

1) Signature-based identification: As we know that different protocols are used different aplications. Each protocol have its own signatures. The signature may includes specific port or a sequence of string or bit sequence. The identification is done byinspecting the application by signature matching in the packet of service flow. So inspection modes adopted by this fingerprint/signature based identification technology can be classified as signature matching at fixed location or random location and status of signature matching. Thus, the DPI can decide to which application, the data flow belongs, for eg. the identification of the $\mathrm{P} 2 \mathrm{P}$ protocol like Bittorrent protocol is performed through the analysis of the peer protocol with reverse engineering method.

2) Application layer based identification: Some services can have in different control and service flows. So in such application former has some features but such features are lacks in later. Hence to identify such service Application gateway based identification is the only choice. This technique first identifies control flow and then relative service flow. This first parse control flow and then service flow in order to define particular protocol. Hence different application gateway is needed Then, Application based on $\mathrm{H} 323$ and SIP protocols are identified by application gate way based technology. These applications negotiate the data channel by exchanging specific signals, like the voice flow encapsulated in the RTP format i.e. the protocol that already establishes such RTP flow cannot be identified through the inspection of the RTP flow only. The protocol can be analyzed only through the proper inspection of the signaling exchange.

3) Behavior-based identification: This type of identification is used only when the data flow cannot be identified according to the protocol by any mean. The behavior-based identification behavior of the user or specific terminal .For eg. SPAM mail data. The SPAM mail only identified by individual behaviors such as mail of lottery and cash prizes.

\section{B. Challenges for DPI}

There are several challenges to build an efficient DPI system. According to AbuHmed et al.[4] the most challenges common are:

1) The false alarms: An Intrusion Detection System (IDS) might generate more than thousands of alerts per day when deployed in any infrastructure. The number of generated alarms that need to be reviewed can escalate rapidly, making the task very difficult to manage. Moreover, due to this, a significant problem is faced by current IDS technology is the high level of false alarms. Snort[5] generates as much as $69 \%$ of false alarms.

2) The complexity of search algorithm: The complexity of an algorithm is important aspect for implementing a signature based systems like deep packet inspection. As most of the time system is busy performing string matching. This is the main concern, as string matching time accounts total share of $40 \%$ to $70 \%$ [6] of the Snort[5] running time i.e. most of time the IDS busy with string matching activity so efficient algorithm is desirable.

3) Rapid growth number of intruder signatures: With advancement in the number and types of attacks, there is a need to define the corresponding intruder signature. Hence, the pace of increasing intruder signature is very high. So the DPI system must be scalable. Snort[5] contains more than 17209 rule sets(as on 25 august 2010) in version 2.8.6. 
4) Overlapping of two or more signature: The most of the false alarms generated $[5,7]$ by snort are basically due to http signature overlapping as there are 1096 rules in http rule set[4].

5) Signatures are in payload: Compared to other IDS/IPS systems the deep packet inspection is more difficult to implement as it examines the whole packet and the pattern of threat/application data is not localized to a particular location. So, the complete payload needs to be inspected against signatures.

6) Coded or Encrypted Data: The data which is encrypted cannot be simply inspected by DPI so, DPI component must be deployed behind some decryption unit.

7) Speed of Line inspection: The current communication systems works at a speed of $10 \mathrm{GbE} / \mathrm{OC} 192$ and approaching to 40GbE/OC768.Hence, the deep packet inspection system need to work at such high speeds otherwise, it might be a bottleneck in the system.

\section{Design Issues}

The DPI systems need to fulfill certain design principles in order to keep in pace with current attack and communication channel speed. These are

1) Availability of signature: The signature must be dynamically updated and must be able to define and defend current threats.

2) Performance and Scalability of system: The hardware based systems, especially ASIC are really fast and can do matching at line speed but the main problem with them is that they are not reconfigurable. In order to update them we need to design chip again and re setup factory to fabricate. Software systems are scalable in nature but need really very fast processor to do operations at line speed of 10GbE/OC192.

3) Space \& Power consumption: The space is key issue in design of DPI, as rapid change in signature might lead to memory scare situation. So the system must be memory efficient. Power consumption also plays a key role when systems are deployed at remote location. Hardware like FPGA is reconfigurable but they consume as much as 40 times power compare to ASIC.

4) Quality of Service issue: DPI needs to address some of the Quality of Service issues like bandwidth management. So it is a pure over head on system to maintain efficient flow of traffic as ISP (internet Service Provider) uses DPI for providing privileged services to their customer. Hence DPI system must provide the support for the same.

\section{EXISTING SOFTWARE BASED TECHNOLOGIES FOR DPI IMPLEMENTATION}

To implement DPI on hardware systems or on software systems we need to develop a process which includes techniques like exact string matching/searching algorithm, regular expressions and a DFA of consisting signatures. Figure 2 shows detailed diagram about relation among them.

Signature consists of sequence of alphabets (string) or regular expressions. Regular expression may represents repetition of characters, special characters, pattern with length constraints and wildcards. Now a day PCRE (Pearl Compactable Regular Expression) is widely used to define the signatures of virus, worms, attacks etc.

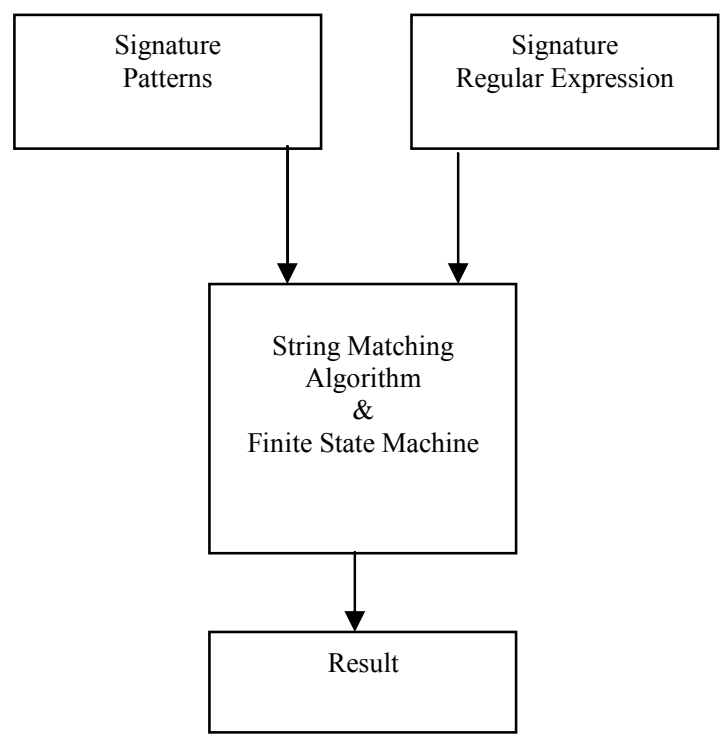

Figure 2: Typical DPI Implementation diagram

Every application has its own signature pattern which may be written in PCRE. Finite state machine are used to represent these signature in either DFA (Deterministic Finite Automaton) or NFA (Nondeterministic Finite Automaton) or lezzy DFA, which is somewhere in between both. Finally signatures matched against packet data (including payload) using pattern matching algorithms. AhoCorasick, Boyer Moore and Wu-Manber are widely used pattern matching algorithms in most of the IDS/IPS and deep packet systems. But there is need of significant changes in implementation techniques of these algorithms to work at such a high speed.

\section{A. Exact string Searching Algorithms}

String matching algorithms can be divided into exact and approximate string matching algorithms. DPI implementation basically depends only on exact string matching algorithm to reduce false alarms. Hence, we cover only exact string matching algorithm in this paper. The exact string matching consists of finding one or more, generally all the occurrences of a string (more generally called a pattern) in a text. The pattern is denoted by $\mathrm{x}=\mathrm{x}[0$.. $\mathrm{m}-1]$; its length is equal to $\mathrm{m}$. The text is denoted by $\mathrm{y}=\mathrm{y}[0 . . \mathrm{n}-1]$; its length is equal to $n$. Both strings are built over a finite set of character called an alphabet denoted by $\sum$ with size equal to $\sigma$. 
One of the most oldest exact string matching algorithm is Aho-Corasick algorithm[8].It is able to find all occurrences of a pattern in a text. It constructs a finite state machine using patterns or keywords. Basic idea behind this algorithm is quite simple. It works as follows: Starting from root node which is the initial state each transaction adds a state to FSM and the result is successful if final accept state or end of pattern is achieved. If in between there is a mismatch, then the failure pointer transfers the state to root or other similar state. Figure 3 illustrates the states of FSM for set of keywords \{hers, his, she, he \}.The Aho-Corasick algorithm is still in use due to its linear time complexity and simplicity in implementing.

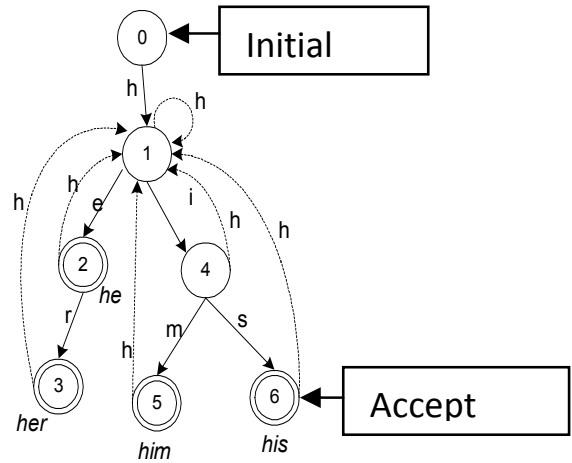

Figure 3: Aho-Corasick State machine

Along with Aho-Corasick other algorithms like Boyer-Moore[9] and Wu-Manber [10] algorithms are used in Intrusion detection/prevention systems like Snort [5, 7] BRO[11],Linux L7-filter[12] with/without modification. The implementation in IDS/IPS is slightly less sophisticated than the original papers describing these algorithms.

The Aho-Corasick algorithm is one of the best algorithm for string matching but it requires large cache for storage. Tuck et al.[13], implement AhoCorasick algorithm with bitmap node compression and path compression to gain compact storage and worst-case performance. They show that the use of such compression techniques they are able to gain almost 50 times in database size reductions on current rule sets. Hence, it can be easily implemented with FPGA, ASIC, and Network Processor as the space required by Aho-Corasick is reduced by remarkable ratio of $50 \%$. There are several variants of BoyerMoore algorithm especially designed for deep packet inspection such as Set-wise Boyer-Moore-Horspool (SBMH) algorithm [14],which was proposed by Fisk and Varghese based on Boyer-Moore-Horspool[15] algorithm which matches the rule set simultaneously. The set of patterns can be compared easily to any position in the text quickly by storing the reversed patterns. Their experiments show that on mediumsized pattern sets, SBHM is faster as compared to both Aho-Corasick and Boyer-Moore algorithms. But the maximum number of shifts is bounded by the LSP (length of the shortest pattern) in the pattern set. Rafiq et al suggest an algorithm [16], with some improvisation over Boyer-Moore algorithm. To performs better in long pattern, long text, and large alphabet set. They suggest two way checking and speed searching phase. As a result the complexity decreases by a considerable value as worst, average and best case complexities are $\mathrm{O}(\mathrm{nm}), \mathrm{O}(\mathrm{n})$ and $\mathrm{O}(\mathrm{n} / \mathrm{m})$ respectively. FNP Algorithm(Fast String Matching) [17] proposed by Tai et al. works well when size of rule sets is less i.e. LSP $<4(35 \%$ of snort[5] rule sets). In such conditions FNP gives better performance as compared to Aho-Corasick, BoyerMoore (SBHM) \& Wu-Manber algorithm. They have shown that the FNP algorithm is very efficient for small LSP regardless of search set size, further same group of author designed FNP2[18], which uses the characteristic of signature rule sets and the hardware facility of Network Processor to maximize performance. They implemented FNP2 on Vitesse IQ2000 Network Processor platform to evaluate the relation between performance and the number of memory accesses for processing multi-pattern matching. Their experimental results reveal that FNP2 is far better than the other algorithms $\{A C$, $\mathrm{SBMH}, \mathrm{E} 2 \mathrm{xB}$ and $\mathrm{MWM}\}$, when the LSP is small. Also this design is able to process L7 payload efficiently when implement on network processor.

There are several other prospect candidate algorithms which may be used with deep packet inspection. A brief summary of such algorithms is described in table $1[19,20]$ and for detailed study visit[20].

\section{B. Regular Expression}

The pure exact string matching is no longer valid in deep packet inspection as new signatures contain

- Wildcards-length restrictions '?' \& '+' notations), dot-star notations (e.g. pattern for Internet radio protocol is "membername. *session. *player" contains two '.*' notation.

- Character ranges-pattern for $\mathrm{ftp}$ protocol is “^220[|x09_lx0d= ]*ftp", contains class inside brackets that includes all the printing characters.

- Counting constraints -bounded repetitions of simple characters, sub-patterns, character set and wildcards.

- Perl compatible regular expressions. 


\begin{tabular}{|c|c|c|c|c|c|}
\hline \multirow[t]{3}{*}{ Algorithm } & \multirow[t]{3}{*}{ Method of Implementing } & \multicolumn{3}{|c|}{ Complexity } & \multirow[t]{3}{*}{ Remarks } \\
\hline & & \multicolumn{2}{|c|}{ Processing } & \multirow{2}{*}{$\begin{array}{c}\text { Searching } \\
\text { time }\end{array}$} & \\
\hline & & Space & Time & & \\
\hline $\begin{array}{l}\text { Aho-Corasick } \\
\text { Algorithm }\end{array}$ & $\begin{array}{l}\text { It is a kind of dictionary-matching algorithm that locates elements of a finite } \\
\text { set of strings (the "dictionary") within an input text. It matches all patterns "at } \\
\text { once", so the complexity of the algorithm is linear in the length of the patterns } \\
\text { plus the length of the searched text plus the number of output matches.[8] }\end{array}$ & $\mathrm{A}: \mathbf{O}(\mathrm{m}+\mathrm{z})$ & $\mathrm{A}: \mathbf{O}(\mathrm{m}+\mathrm{z})$ & $\mathrm{A}: \mathbf{O}(\mathrm{n})$ & $\begin{array}{l}\text { There can be a quadratic } \\
\text { number of matches if every } \\
\text { substring matches }\end{array}$ \\
\hline $\begin{array}{l}\text { Wu-Manber } \\
\text { Algorithm }\end{array}$ & $\begin{array}{l}\text { Use Hash Based Searching. A Variant of Boyer-Moore Algorithm. Use the } \\
\text { concept of skipping characters and also find the candidate for matching. }\end{array}$ & $\mathrm{A}: \mathbf{O}(\mathrm{m}+\mathrm{z})$ & $\mathrm{A}: \mathbf{O}(\mathrm{m}+\mathrm{z})$ & $\mathrm{A}: \mathbf{O}(\mathrm{Bn} / \mathrm{m})$ & $\begin{array}{l}\text { Fast Searching algorithm for } \\
\text { large pattern searching. }\end{array}$ \\
\hline $\begin{array}{l}\text { Boyer-Moore } \\
\text { algorithm }\end{array}$ & $\begin{array}{l}\text { The Boyer-Moore algorithm is one of the most efficient string-matching } \\
\text { algorithm for such applications. it process the pattern from right to left and if } \\
\text { a mismatch (or a complete match of the complete pattern) is occurs it uses } \\
\text { two precomputed functions to shift the window to the right. The two shift } \\
\text { functions which used by it are called the good-suffix shift (or matching } \\
\text { shift)and the bad-character shift. [9] }\end{array}$ & $\mathrm{A}: \mathbf{O}(\mathrm{m}+\sigma)$ & $\mathrm{A}: \mathbf{O}(\mathrm{m}+\sigma)$ & $\mathrm{A}: \mathbf{O}(\mathrm{mn})$ & $\begin{array}{l}\text { text character } \\
\text { comparison is as follows: } \\
\text { W: } \boldsymbol{O}(3 \mathrm{n}) \\
\text { B: } \boldsymbol{O}(\mathrm{n} / \mathrm{m})\end{array}$ \\
\hline $\begin{array}{l}\text { Turbo-Boyer } \\
\text { Moore } \\
\text { algorithm }\end{array}$ & $\begin{array}{l}\text { The Turbo-BM algorithm is a refined version of the Boyer-Moore algorithm. } \\
\text { there is no need for extra preprocessing and only constant extra space is } \\
\text { needed as compare to the original one. It remembers text patterns for } \\
\text { matching.[21] }\end{array}$ & $\mathrm{A}: \mathbf{O}(\mathrm{m}+\sigma)$ & $\mathrm{A}: \mathbf{O}(\mathrm{m}+\sigma)$ & $\mathrm{A}: \mathbf{O}(\mathrm{n})$ & $\begin{array}{l}\text { text character } \\
\text { comparison is as follows: } \\
\text { W: } \boldsymbol{O}(2 \mathrm{n})\end{array}$ \\
\hline $\begin{array}{l}\text { Apostolico- } \\
\text { Giancarlo } \\
\text { algorithm }\end{array}$ & $\begin{array}{l}\text { Improvised version of boyer_moore. It uses SKIP table to store the } \\
\text { information about the longest suffix of the pattern ending at the right position } \\
\text { of the window for that matching attemps. These information are stored in a } \\
\text { table skip.[22] }\end{array}$ & $\mathrm{A}: \mathbf{O}(\mathrm{m}+\sigma)$ & $\mathrm{A}: \mathbf{O}(\mathrm{m}+\sigma)$ & A: $\mathbf{O}(\mathrm{n})$ & $\begin{array}{l}\text { text character } \\
\text { comparison is as follows: } \\
\text { W: } \boldsymbol{O}(3 / 2 \mathrm{n})\end{array}$ \\
\hline $\begin{array}{c}\text { Reverse } \\
\text { Colussi } \\
\text { algorithm }\end{array}$ & $\begin{array}{l}\text { Again the variant of Boyer-Moore algorithm, It uses a hash table and } \\
\text { partitions the set of pattern positions into two different and disjoint } \\
\text { subsets.[23] }\end{array}$ & $\mathrm{A}: \mathbf{O}\left(\mathrm{m}^{2}\right)$ & $\mathrm{A}: \mathbf{O}(\mathrm{m} \sigma)$ & $\mathrm{A}: \mathbf{O}(\mathrm{n})$ & $\begin{array}{l}\text { text character comparison } \\
\text { is }: \mathrm{W}: \boldsymbol{O}(2 \mathrm{n})\end{array}$ \\
\hline $\begin{array}{l}\text { Horspool } \\
\text { algorithm }\end{array}$ & $\begin{array}{l}\text { Less complicated version of well known Boyer-Moore.In order to compute } \\
\text { the shifts in the Boyer-Moore algorithm it uses only the bad-character shifts } \\
\text { of the rightmost character of the window.[15] }\end{array}$ & $\mathrm{A}: \mathbf{O}(\mathrm{m}+\sigma)$ & $\mathrm{A}: \mathbf{O}(\sigma)$ & A: $\mathbf{O}(\mathrm{mn})$ & $\begin{array}{l}\text { text character comparison } \\
\text { is as follows: } \\
\text { A:between } \boldsymbol{O}(1 / \sigma) \text { to } \boldsymbol{O}(2 /( \\
+1))\end{array}$ \\
\hline $\begin{array}{l}\text { Quick Search } \\
\text { algorithm }\end{array}$ & $\begin{array}{l}\text { Easiest implementation of BM algorithm and works only on bad-character } \\
\text { shift table. This is very efficient algorithm we have short pattern and large } \\
\text { text to be searched.[24] }\end{array}$ & $\mathrm{A}: \mathbf{O}(\mathrm{m}+\sigma)$ & $\mathrm{A}: \mathbf{O}(\sigma)$ & A: $\mathbf{O}(m n)$ & $\begin{array}{l}\text { very fast for short patterns } \\
\text { and large alphabets. }\end{array}$ \\
\hline $\begin{array}{l}\text { Optimal } \\
\text { Mismatch } \\
\text { algorithm }\end{array}$ & $\begin{array}{l}\text { Another version of simplified Quick Search algorithm, It is efficient when } \\
\text { patterns are searched from least frequent to most frequent one.[24] }\end{array}$ & A: $\mathbf{O}\left(m^{2}+\sigma\right)$ & $\mathrm{A}: \mathbf{O}(\mathrm{m}+\sigma)$ & $\mathrm{A}: \mathbf{O}(\mathrm{mn})$ & $\begin{array}{l}\text { frequencies of each of the } \\
\text { character of the alphabet } \\
\text { must available in advance. }\end{array}$ \\
\hline $\begin{array}{c}\text { Maximal } \\
\text { Shift } \\
\text { algorithm }\end{array}$ & $\begin{array}{l}\text { Another implementation of optimal mismatch algorithm in this case } \\
\text { characters are scanned from the larger shift first before the shorter shift. This } \\
\text { help to maximize the length of each shift.[24] }\end{array}$ & A: $\mathbf{O}\left(m^{2}+\sigma\right)$ & $\mathrm{A}: \mathbf{O}(\mathrm{m}+\sigma)$ & $\mathrm{A}: \mathbf{O}(\mathrm{mn})$ & $\begin{array}{l}\text { quadratic worst case time } \\
\text { complexity }\end{array}$ \\
\hline $\begin{array}{l}\text { Zhu-Takaoka } \\
\text { algorithm }\end{array}$ & $\begin{array}{l}\text { Simplification version of well known Boyer-Moore algorithm, It uses the } \\
\text { technique of two consecutive text characters in order to compute the bad- } \\
\text { character shift[25] }\end{array}$ & $\mathrm{A}: \mathbf{O}\left(\mathrm{m}+\sigma^{2}\right)$ & $\mathrm{A}: \mathbf{O}\left(\mathrm{m}+\sigma^{2}\right)$ & $\mathrm{A}: \mathbf{O}(\mathrm{mn})$ & $\begin{array}{l}\text { The searching phase has } \\
\text { a quadratic worst case. }\end{array}$ \\
\hline $\begin{array}{l}\text { Berry- } \\
\text { Ravindran } \\
\text { algorithm }\end{array}$ & $\begin{array}{l}\text { Uses the best of Quick Search and Zhu and Takaoka algorithms. It shifts } \\
\text { pattern based on two consecutive text characters from the right side of pattern } \\
\text { window[26]. }\end{array}$ & A: $\mathbf{O}\left(m+\sigma^{2}\right)$ & A: $\mathbf{O}\left(m+\sigma^{2}\right)$ & $\mathrm{A}: \mathbf{O}(\mathrm{mn})$ & \\
\hline $\begin{array}{l}\text { Smith } \\
\text { algorithm }\end{array}$ & $\begin{array}{l}\text { It based on the concept which uses maximum of the Horspool bad-character } \\
\text { shift function and the Quick Search bad-character shift function.[27] }\end{array}$ & $\mathrm{A}: \mathbf{O}(\sigma)$ & $\mathrm{A}: \mathbf{O}(\mathrm{m}+\sigma)$ & $\mathrm{A}: \mathbf{O}(\mathrm{mn})$ & $\begin{array}{l}\text { The searching phase may } \\
\text { lead to quadratic worst case } \\
\text { time complexity. }\end{array}$ \\
\hline $\begin{array}{c}\text { Raita } \\
\text { algorithm }\end{array}$ & $\begin{array}{l}\text { First compares the character patterns in order of last, then the first and finally } \\
\text { the middle then move on the other pattern. Shift concept is just like that of } \\
\text { Horspool algorithm[28] }\end{array}$ & $\mathrm{A}: \mathbf{O}(\sigma)$ & $\mathrm{A}: \mathbf{O}(\mathrm{m}+\sigma)$ & $\mathrm{A}: \mathbf{O}(\mathrm{mn})$ & $\begin{array}{l}\text { The searching phase has } \\
\text { a quadratic worst case time } \\
\text { complexity. }\end{array}$ \\
\hline $\begin{array}{l}\text { Brute force } \\
\text { algorithm }\end{array}$ & $\begin{array}{l}\text { Searches for pattern in every position in text between } 0 \text { and }(\mathrm{n}-\mathrm{m}) \text { and shifts } \\
\text { pattern by one position to the right after each attempt till found exact } \\
\text { matching or reaches end of pattern. } \\
\text { The expected number of text character comparisons is } 2 \mathrm{n} \text {. }\end{array}$ & $\begin{array}{c}\text { Not } \\
\text { Required }\end{array}$ & $\begin{array}{l}\text { Not } \\
\text { Required }\end{array}$ & $\begin{array}{l}\text { A: } \mathbf{O}(\mathrm{mn}) \\
(\mathrm{W}, \mathrm{B}, \mathrm{A})\end{array}$ & $\begin{array}{l}\text { 1. Constant extra space } \\
\text { needed. } \\
2 . \text { Comparisons can be } \\
\text { done in any order. }\end{array}$ \\
\hline $\begin{array}{l}\text { Automaton } \\
\text { based } \\
\text { Algorithm }\end{array}$ & $\begin{array}{l}\text { Based on the principle of DFA.In order to search it need to builds the a } \\
\text { minimal deterministic automaton which recognizing the language } \sum^{*} x ;[29]\end{array}$ & $\mathrm{A}: \mathbf{O}(\mathrm{m} \sigma)$ & $\mathrm{A}: \mathbf{O}(\mathrm{m} \sigma)$ & $\begin{array}{c}\mathrm{A}: \mathbf{O}(\mathrm{n}) \\
\mathrm{W}: \mathbf{O}(\mathrm{n} \log (\sigma))\end{array}$ & $\begin{array}{l}\text { Direct access table } \\
\text { complexity increase by } \\
\text { W:O(nlog( }(\sigma))\end{array}$ \\
\hline $\begin{array}{l}\text { Karp-Rabin } \\
\text { algorithm }\end{array}$ & $\begin{array}{l}\text { Uses hashing technique for preprocessing in order to reduce quadratic time } \\
\text { complexity[30] }\end{array}$ & $\begin{array}{c}\text { Constant } \\
\text { space }\end{array}$ & $\mathrm{A}: \mathbf{O}(\mathrm{m})$ & $\mathrm{A}: \mathbf{O}(\mathrm{mn})$ & $\begin{array}{l}\text { expected running time } \\
\mathrm{W}: \mathbf{O}(\mathrm{n}+\mathrm{m})\end{array}$ \\
\hline $\begin{array}{l}\text { Shift Or } \\
\text { algorithm }\end{array}$ & $\begin{array}{l}\text { Based on bitwise technique. It is an efficient algorithm if pattern size less than } \\
\text { the memory-word size of the machine. Good for approx string matching. [31] }\end{array}$ & A: $\mathbf{O}(m+\sigma)$ & $\mathrm{A}: \mathbf{O}(\mathrm{m}+\sigma)$ & $\mathrm{A}: \mathbf{O}(\mathrm{n})$ & $\begin{array}{l}\text { Independent from the } \\
\text { alphabet size and the } \\
\text { pattern length }\end{array}$ \\
\hline
\end{tabular}




\begin{tabular}{|c|c|c|c|c|c|}
\hline $\begin{array}{l}\text { Morris-Pratt } \\
\text { algorithm }\end{array}$ & $\begin{array}{l}\text { It follows a tight analysis of the Brute Force algorithm from left to right, and } \\
\text { Remembers information gathered during the scan of the text i.e. the matched } \\
\text { portion of text and pattern.[32] }\end{array}$ & $\mathrm{A}: \mathbf{O}(\mathrm{m})$ & $\mathrm{A}: \mathbf{O}(\mathrm{m})$ & $\mathrm{A}: \mathbf{O}(\mathrm{n}+\mathrm{m})$ & $\begin{array}{l}\text { Max. delay bounded by } \\
\text { m. }\end{array}$ \\
\hline $\begin{array}{l}\text { Knuth- } \\
\text { Morris-Pratt } \\
\text { algorithm }\end{array}$ & $\begin{array}{l}\text { Follow the tight analysis of Morris-Pratt and do comparisons from left to } \\
\text { right. It is a simplified and improvised version of Morris-Pratt Algorithm.[33] }\end{array}$ & $\mathrm{A}: \boldsymbol{O}(\mathrm{m})$ & A: $\boldsymbol{O}(\mathrm{m})$ & $\mathrm{A}: \boldsymbol{O}(\mathrm{n}+\mathrm{m})$ & $\begin{array}{l}\text { Max delay } \log \Phi(\mathrm{m}) \\
\text { where } \Phi \text { is the golden } \\
\operatorname{ratio}(\Phi=(1+\sqrt{5}) / 2)\end{array}$ \\
\hline $\begin{array}{l}\text { Simon } \\
\text { algorithm }\end{array}$ & $\begin{array}{l}\text { It reduce the size of Automata which helps in economical implementation of } \\
\qquad \mathrm{A}(\mathrm{x}) \text { the minimal DFA recognizing } \sum^{*} \mathrm{x} .[34]\end{array}$ & $\mathrm{A}: \mathbf{O}(\mathrm{m})$ & $\mathrm{A}: \mathbf{O}(\mathrm{m})$ & $\mathrm{A}: \mathbf{O}(\mathrm{m}+\mathrm{n})$ & $\begin{array}{l}\text { Max. delay bounded by } \\
\min \{1+\log 2 \mathrm{~m}, \sigma\}\end{array}$ \\
\hline $\begin{array}{l}\text { Colussi } \\
\text { algorithm }\end{array}$ & $\begin{array}{l}\text { Simplified and improved version of Knuth-Morris-Pratt algorithm. The set of } \\
\text { pattern positions are partitioned in two disjoint subsets; the first is scanned } \\
\text { from left to right and if it matches then the second subset is scanned from } \\
\text { right to left.[35] }\end{array}$ & $\mathrm{A}: \mathbf{O}(\mathrm{m})$ & $\mathrm{A}: \mathbf{O}(\mathrm{m})$ & $\mathrm{A}: \mathbf{O}(\mathrm{m})$ & $\begin{array}{l}\text { Text character } \\
\text { comparisons } \\
\mathrm{W}: \mathbf{O}(2 / 3 n)\end{array}$ \\
\hline $\begin{array}{l}\text { Galil- } \\
\text { Giancarlo } \\
\text { algorithm }\end{array}$ & Simplified and improved implementation of Colussi algorithm[36] & $\mathrm{A}: \mathbf{O}(\mathrm{m})$ & $\mathrm{A}: \mathbf{O}(\mathrm{m})$ & $\mathrm{A}: \mathbf{O}(\mathrm{m})$ & $\begin{array}{l}\text { Text character } \\
\text { comparisons } \\
\mathrm{W}: \mathbf{O}(4 / 3 \mathrm{n})\end{array}$ \\
\hline $\begin{array}{l}\text { Not So Naive } \\
\text { algorithm }\end{array}$ & $\begin{array}{l}\text { In searching phase the character comparisons are made with the pattern } \\
\text { positions in the following order } 1,2, \ldots, \mathrm{m}-2, \mathrm{~m}-1,0 .[38]\end{array}$ & $\begin{array}{l}\text { Constant } \\
\text { space }\end{array}$ & $\begin{array}{l}\text { Constant } \\
\text { time }\end{array}$ & $\mathrm{A}: \mathbf{O}(\mathrm{nm})$ & $\begin{array}{l}\text { preprocessing phase in } \\
\text { constant time and space }\end{array}$ \\
\hline $\begin{array}{l}\text { Reverse } \\
\text { Factor }\end{array}$ & $\begin{array}{c}\text { It uses suffix automaton of } x^{R} \text { or DAWG for Directed Acyclic Word Graph) } \\
\text { in order to made some prefix scanning from left to right hence improves the } \\
\text { length of the shift. It is fast on practice for long patterns and small } \\
\text { alphabets.[39] }\end{array}$ & $\mathrm{A}: \mathbf{O}(\mathrm{m})$ & $\mathrm{A}: \mathbf{O}(\mathrm{m})$ & $\mathrm{A}: \mathbf{O}(\mathrm{nm})$ & Optimal in the average. \\
\hline $\begin{array}{l}\text { Turbo RF } \\
\text { algorithm }\end{array}$ & $\begin{array}{l}\text { Simplified and refinement implementation of the Reverse Factor } \\
\text { algorithm. }[40]\end{array}$ & $\mathrm{A}: \mathbf{O}(\mathrm{m})$ & $\mathrm{A}: \mathbf{O}(\mathrm{m})$ & $\mathrm{A}: \mathbf{O}(\mathrm{n})$ & $\begin{array}{l}\text { text character } \\
\text { comparison is } \mathrm{W}: \mathbf{O}(2 \mathrm{n})\end{array}$ \\
\hline $\begin{array}{l}\text { Forward } \\
\text { Dawg } \\
\text { Matching } \\
\text { algorithm }\end{array}$ & $\begin{array}{l}\text { It uses the smallest suffix automaton (also called DAWG for Directed Acyclic } \\
\text { Word Graph) of the pattern to computes the longest factor of the pattern } \\
\text { ending at each position in the text..[41] }\end{array}$ & NA & NA & $\mathrm{W}: \mathbf{O}(\mathrm{n})$ & $\begin{array}{l}\text { text character } \\
\text { comparison is as follows: } \\
\mathrm{W}: \mathbf{O}(\mathrm{n})\end{array}$ \\
\hline $\begin{array}{l}\text { Backward } \\
\text { Oracle } \\
\text { Matching }\end{array}$ & $\begin{array}{l}\text { It uses the suffix oracle of } x^{R} \text { instead of the suffix automaton of } x^{R} \text {. used in } \\
\text { the Reverse Factor algorithm Efficient in long patterns and small alphabets } \\
\text { sets.[43] }\end{array}$ & $\mathrm{A}: \mathbf{O}(\mathrm{m})$ & $\mathrm{A}: \mathbf{O}(\mathrm{m})$ & $\mathrm{A}: \mathbf{O}(\mathrm{nm})$ & Optimal in the average. \\
\hline Galil-Seiferas & $\begin{array}{l}\text { Uses a decomposition technique for preprocessing. constant extra space } \\
\text { complexity.[44] }\end{array}$ & $\begin{array}{l}\text { Constant } \\
\text { space }\end{array}$ & $\mathrm{A}: \mathbf{O}(\mathrm{m})$ & $\mathrm{A}: \mathbf{O}(\mathrm{n})$ & $\begin{array}{l}\text { text character } \\
\text { comparison is } \mathrm{W}: \boldsymbol{O}(5 \mathrm{n})\end{array}$ \\
\hline $\begin{array}{l}\text { Two Way } \\
\text { algorithm }\end{array}$ & $\begin{array}{l}\text { Prerequisite of order alphabet. Divides pattern into two sub-strings for pattern } \\
\text { matching... } 45]\end{array}$ & $\begin{array}{c}\text { Constant } \\
\text { space }\end{array}$ & $\mathrm{A}: \mathbf{O}(\mathrm{m})$ & $\mathrm{A}: \mathbf{O}(\mathrm{n})$ & $\begin{array}{l}\text { text character } \\
\text { comparison is } \\
\text { W: } \boldsymbol{O}(2 \mathrm{n}-\mathrm{m})\end{array}$ \\
\hline $\begin{array}{l}\text { Skip Search } \\
\text { algorithm }\end{array}$ & $\begin{array}{l}\text { Uses buckets of positions for each character of the alphabet. For each } \\
\text { character of the alphabet, a bucket collects all the positions of that character in } \\
\text { pattern x.[46] }\end{array}$ & $\mathrm{A}: \mathbf{O}(\mathrm{m}+\sigma)$ & $\mathrm{A}: \mathbf{O}(\mathrm{m}+\sigma)$ & $\mathrm{A}: \mathbf{O}(\mathrm{nm})$ & $\begin{array}{l}\text { text character } \\
\text { comparison is }: \mathrm{W}: \boldsymbol{O}(\mathrm{n})\end{array}$ \\
\hline $\begin{array}{l}\text { KMP Skip } \\
\text { Search }\end{array}$ & $\begin{array}{c}\text { Refined version of the Skip Search algorithm. It is linear Skip Search by } \\
\text { using the two shift tables of Morris-Pratt and KMP .[46] }\end{array}$ & $\mathrm{A}: \mathbf{O}(\mathrm{m}+\sigma)$ & $\mathrm{A}: \mathbf{O}(\mathrm{m}+\sigma)$ & $\mathrm{A}: \mathbf{O}(\mathrm{n})$ & $\begin{array}{l}\text { text character } \\
\text { comparison is: } \mathrm{W}: \boldsymbol{O}(\mathrm{n})\end{array}$ \\
\hline $\begin{array}{l}\text { Alpha Skip } \\
\text { Search } \\
\text { algorithm }\end{array}$ & $\begin{array}{l}\text { Refinement of the Skip Search algorithm uses buckets of positions for each } \\
\text { factor of length } \log \sigma(\mathrm{m}) \text { of the each pattern. }\end{array}$ & $\mathrm{A}: \mathbf{O}(\mathrm{m})$ & $\mathrm{A}: \mathbf{O}(\mathrm{m})$ & $\mathrm{A}: \mathbf{O}(\mathrm{nm})$ & $\begin{array}{l}\text { text character } \\
\text { comparison is as follows: } \\
W: \boldsymbol{O}(\log \sigma(m) .(n /(m- \\
\qquad \log \sigma(m))))\end{array}$ \\
\hline
\end{tabular}
W: Worst case
$m$ : Length of pattern
A: Average Case
$n$ :length of text

$z: z$ is the number of pattern occurrences

\section{B: Best case}

$\sigma:$ Size of alphabet (a finite set of character)

NA: No description available in literature 
To accommodate these patterns large data structures up to few gigabytes are needed. The signatures contain more complex and general patterns; therefore, the exact string matching algorithm cannot perform optimally. In [47] the authors design a regular expression model and try to develop an evolution framework for regular expression data structure. Table 2 [48] compares the various regular expression used in snort, Linux L-7 filter.

TABLE 2: COMPARISON OF REGULAR EXPRESSION IN NETWORKING APPLICATION[48]

\begin{tabular}{|l|l|l|}
\hline \multicolumn{1}{|c|}{ RegEx } & \multicolumn{1}{|c|}{ SNORT } & \multicolumn{1}{|c|}{ L7-Filter } \\
\hline \# of regular expressions analyzed & 1555 & 70 \\
\hline$\%$ of pattern starting with “^” & $74.4 \%$ & $72.8 \%$ \\
\hline $\begin{array}{l}\text { \% of pattern starting with wildcards } \\
\text {., +, ?, *” }\end{array}$ & $74.9 \%$ & $75.7 \%$ \\
\hline Avg. \# wildcards per pattern & 4.65 & 1.03 \\
\hline$\%$ of pattern with class "[]" & $31.6 \%$ & $52.8 \%$ \\
\hline Avg. \# class per pattern & 7.97 & 4.78 \\
\hline $\begin{array}{l}\text { \% of pattern with length } \\
\text { restrictions on class and wildcards }\end{array}$ & $56.3 \%$ & $21.4 \%$ \\
\hline
\end{tabular}

To implement the regular expression, Finite State Machine is best choice.

\section{Finite State Machine}

The main task of pattern matching in deep packet inspection consists of comparing a large number of static signatures (patterns) against stream of data at line speed (10GbE) using String Searching algorithm. The main design approaches that have been described are String Tree (brute-force) and finite state machine (FSM). The String Tree (brute-force) approach performs the full parallel pattern comparison against data stream in each clock cycle. Since no record about matched states is maintained across cycles, hence many extra character comparators are required to implement simple regular expressions. It is practically not feasible, to implement complete rule sets using string tree due to its large size and more computational time. There two types of finite state machine Non deterministic Finite Automata (NFA) and Deterministic Finite Automata (DFA). NFA has one or more final states corresponding to single initial state. Edges can be labeled with null or single characters which mean simultaneously there are multiple states active at a time.NFA is a very useful tool for parallel processing so it can be used in DPI there are several efforts of constructing NFA based DPI systems. In[49], clark et al. proposed an FPGA based design which proved that single FPGA is able to do matching at network speeds up to $10 \mathrm{Gbps}$. It is scalable to perform large-scale pattern matching at the edge of the network, in devices such as routers, switches, and network interface cards. Multi-FPGA systems which are capable of operating at backbone router rates. This type of system enables deep packet inspection to ensure QoS issue and Surveillance before actually packet is received at end host. Another design suggested by Sidhu et al.[50], present an FPGA and
SRGA based NFA which is capable of comparing more than 1,500 patterns ( $>17,000$ characters) against an input stream using a low-end Xilinx Virtex-1000 FPGA at a line speed of over $800 \mathrm{Mbps}$.

DFA is widely used to perform regular expression matching in linear time. In DFA processing an input string involves one DFA state traversal per character, which translates into a deterministic number of memory accesses so it take linear time. DFAs have a property of predictable bandwidth requirement as a result in recent researchers tries to exploit this capability to achieve optimal solutions using DFA. $\operatorname{In}[48]$ researcher have tried to rewrite rules and selective grouping of multiple patterns to achieve low memory consumptions as compare to traditional DFA(4312 KB against $7628 \mathrm{~KB}$ of traditional DFA). Michela et al.[51] tries to modify DFA to build hybridFA to achieve modest memory requirement comparable to NFA and average case memory bandwidth requirement similar to DFA. They are able to achieve worst case memory bandwidth linear to the number of regular expression containing counting constraints and dot start notation irrespective of number of states in automata. Split-AC[52] is another version of DFA based Aho-Corasick algorithm which is small in term of memory footprint of AC Algorithm but is unable to achieve desirable computational speed. S. kumar et al. proposed Delayed Input DFA(D2FA)[53] with the concept of default transactions used to ensure largest space reduction. Using D2FA reduces memory space requirements by more than $95 \%$. Hence the entire automata can be embedded on a single chip and it supports several memory accesses per input characters. With D2FA a worst case throughput of OC192 is achievable executing several thousand expressions simultaneously. Continuing their work from D2FA, S. Kumar et al.[54] proposed CD2FA (Content Addressed Delayed Input DFA) where the states of D2FA labeled with content labels. Content labels help to reduce default transaction to achieve higher throughput and scalability in number of rules as compared to DFA. Portable predictive Pattern Matching Finite State Machine (P3FSM)[55], yet another version of DFA proposed by Vespa et al. which is a software based FSM but able to achieve throughput at par to hardware based pattern matching with SDFA and CA technique. SDFA(Split-DFA) eliminates redundant transactions, then partitions the DFA into multiple blocks called primary and secondary blocks and CA(character aware) which takes account of distribution of characters which helps FSM quickly isolate the correct next state based on incoming packet characters. In another work S. Kumar et al.[56], suggest three key issues with traditional DFAs as Insomnia, Amnesia, and Acalculia. Insomnia occurs when the signature is not matched completely i.e. the tail portion of signature is isolated from the automaton. Amnesia is a problem due to DFA's limited memory as it is only able to remember a single state of parsing and ignoring everything about earlier parse and associated partial matches. Acalculia occurs 
due to inability of DFA or NFA to count occurrence of certain sub-expression in input stream. They suggest some curing methodologies to achieve reduction in memory requirement of a state-of-the-art regular expressions implementation by up to 100 times as well as 2 to 3 fold increase in packet throughput. $\operatorname{In}[57]$, Smith et al.try to solve the state space explosion problem of DFA. They designed XFA(extended Finite Automaton) which helps to improve performance and decrease per flow state which cause state space explosion. In table 3 we show comparisons of DFA and NFA adopted from Fang et al.[48]

TABLE 3: WORST CASE COMPARISONS OF DFA AND NFA[48]

\begin{tabular}{|c|c|c|c|c|}
\hline \multirow{2}{*}{$\begin{array}{c}\text { Finite } \\
\text { State } \\
\text { Machine }\end{array}$} & \multicolumn{2}{|c|}{$\begin{array}{c}\text { On regular expression } \\
\text { of length } \mathbf{n}\end{array}$} & \multicolumn{2}{c|}{$\begin{array}{c}\text { M regular expression } \\
\text { compiled together }\end{array}$} \\
\cline { 2 - 5 } & $\begin{array}{c}\text { Processing } \\
\text { Complexity }\end{array}$ & $\begin{array}{c}\text { Storage } \\
\text { Cost }\end{array}$ & $\begin{array}{c}\text { Processing } \\
\text { Complexity }\end{array}$ & $\begin{array}{c}\text { Storage } \\
\text { Cost }\end{array}$ \\
\hline NFA & $\mathbf{O}\left(\mathrm{n}^{2}\right)$ & $\mathbf{O}(\mathrm{n})$ & $\mathbf{O}\left(\mathrm{n}^{2} \mathrm{~m}\right)$ & $\mathbf{O}(\mathrm{nm})$ \\
\hline DFA & $\mathbf{O}(1)$ & $\mathbf{O}\left(\sum^{\mathrm{n}}\right)$ & $\mathbf{O}(1)$ & $\mathbf{O}\left(\sum^{\mathrm{nm}}\right)$ \\
\hline
\end{tabular}

$\sum$ : finite set of Input symbols/ n, m: length of expression

\section{V.RESEARCH GAPS}

The main issues with existing implementations is that there is not a single implementation which is efficient in term of space, high throughput, high accuracy and low false alarm when implemented on high speed network like 10GbE/OC192 link.

\section{VI.PROPOSED IMPLEMENTATION USING SOFT COMPUTING TECHNIQUES.}

So far we have studied string matching algorithms, regular expression and finite state machine as tools for DPI. Sometimes hashing is also used with these techniques.. In this section we propose soft computing technique to fill research gaps. Soft computing is gaining popularity in recent time due to its properties like vagueness, self learning, adaptive, and ability to process noisy data well. There are several soft computing technique like fuzzy logic, artificial intelligence, expert system, genetic algorithm etc. Out of these technique fuzzy logic, expert system and neural networks can be implemented in DPI to refine the overall process. One of the functions of deep packet inspection is traffic shaping by ISP for that hybrid techniques like Fuzzy ARTMAP (Hybrid Neural net and Fuzzy Logic) can be used. As recently researchers[58] proved that it can be used to describe peer-to-peer traffics in internet. Fuzzy ARTMAP can be efficiently used in other DPI applications like QoS and network surveillance. Fuzzy ARTMAP is capable in reduce false detection rate and provide high accuracy and low false alarms due to its property of processing noisy data, incremental learning capability and vagueness. It is easy to embed fuzzy ARTMAP to the backbone routers and allows continuous running in background because most of DPI based filters deployed at gateways as it consumes relatively low space as compare to FSM. The Fuzzy ARTMAP or other technique of neural network can be implemented on the results of string matching algorithms. Figure 4 shows the flowchart of typical DPI system with embedded AI Technique.

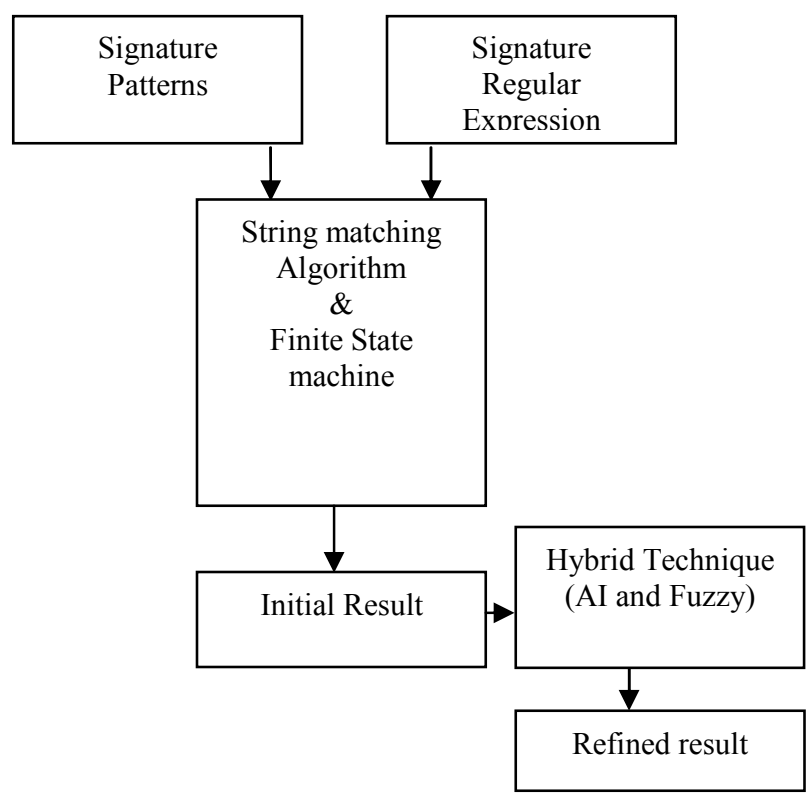

Figure 4: Typical DPI Implementation graph with AI techniques

\section{CONCLUSIONS}

In this paper we have pointed out various software aspects of Deep Packet Inspection technique. Deep packet inspection is implemented on payload against large set of signatures. This requires efficient string matching algorithms. Several authors tried to improve existing algorithms to fit into DPI environment but at 10GbE/OC192 (Approaching 40GbE/OC768) line speed we still need a really very fast algorithm. Regular Expressions are integral part of signatures, but they consume lots of space when implemented in Finite State machine (FSM). FSM like DFA and NFA used to implement the signature on hardware or on software. The main problem with DFA is state space explosion with large signature set. Hence, in future memory efficient DFA is still needed. This work proposed hybrid techniques like fuzzy ARTMAP which are really powerful due to their properties and are able to provide high throughput, high accuracy, and low false alarm at a speed of 10GbE/OC192 link.

\section{REFERENCES}

[1] R. Bendrath, "Global technology trends and national regulation: Explaining Variation in the Governance of Deep Packet Inspection " in International Studies Annual Convention New York City, 2009.

[2] Esoft, "Modern Network Security: The Migration to Deep Packet Inspection," 2005.

[3] huawei, "The DPI Technology and Functions," http://www.huawei.com/products/datacomm/catalog.do?id=12 $35,2010$.

[4] T. AbuHmed, A. Mohaisen, and D. H. Nyang, "Deep Packet Inspection for Intrusion Detection Systems: A Survey," Technical Report,Security Research Laboratory, Inha University, Information Incheon 402-751, Korea 2007.

[5] SNORT, "Network intrusion detection system," Available: www.snort.org. 
[6] A. Spyros, G. A. Kostas, and P. M. Evangelos, "Generating realistic workloads for network intrusion detection systems," in Proceedings of the 4th international workshop on Software and performance Redwood Shores, California: ACM, 2004, pp. 207-251.

[7] M. Roesch, "Snort-lightweight intrusion detection for networks," in Proceedings of LISA'99: 13th Systems Administration Conference, 1999, pp. 229-238.

[8] A. V. Aho and M. J. Corasick, "Efficient string matching: an aid to bibliographic search," Communications of the ACM, vol. 18 , p. 340,1975

[9] R. S. Boyer and J. S. Moore, "A fast string searching algorithm," Commun. ACM, vol. 20, pp. 762-772, 1977.

[10] S. Wu and U. Manber, "A fast algorithm for multi-pattern searching," Technical Report TR-94-17, Department of Computer Science,University of Arizona, 1994.

[11] I. D. S. Bro, "Homepage: http://www.bro-ids.org," Sept., 2010

[12] L.-f. c. p. team, "L7-filter Classifier." vol. [Accessed: Sept.,2010]: Available:http://17-filter.sourceforge.net/, 2010.

[13] N. Tuck, T. Sherwood, B. Calder, and G. Varghese, "Deterministic memory-efficient string matching algorithms for intrusion detection," in INFOCOM 2004. Twenty-third AnnualJoint Conference of the IEEE Computer and Communications Societies, 2004, pp. 2628-2639 vol.4.

[14] M. Fisk and G. Varghese, "An analysis of fast string matching applied to content-based forwarding and intrusion detection," Technical Report CS2001-0670 (updated version), University of California - San Diego, 2002.

[15] R. N. Horspool, "Practical fast searching in strings," Software: Practice and Experience, vol. 10, pp. 501-506, 1980.

[16] A. N. M. E. Rafiq, M. W. El-Kharashi, and F. Gebali, "A fast string search algorithm for computer networking," in IEEE Pacific Rim Conference on Communications, Computers and signal Processing, 2003., 2003, pp. 764-767 vol.2.

[17] L. Rong-Tai, H. Nen-Fu, K. Chia-Nan, and C. Chih-Hao, "A fast pattern matching algorithm for network processor-based intrusion detection system," in 2004 IEEE International Conference on Performance, Computing, and Communications, 2004, pp. 271-275.

[18] L. Rong-Tai, H. Nen-Fu, K. Chia-Nan, C. Chih-Hao, and C. Chi-Chieh, "A fast pattern-match engine for network processor-based network intrusion detection system," in Proceedings. ITCC 2004. International Conference on Information Technology: Coding and Computing, 2004., 2004, pp. 97-101 Vol.1.

[19] A. N. M. Rafiq, M. W. El-Kharashi, and F. Gebali, "A fast string search algorithm for deep packet classification," Computer Communications, vol. 27, pp. 1524-1538, 2004.

[20] C. Charras and T. Lecroq, "EXACT STRING MATCHING ALGORITHMS." vol. sept.,29,2010: Laboratoire d'Informatique de Rouen,Université de Rouen,Faculté des Sciences et des Techniques, pp. [Online].Available: http://www-igm.univ-mlv.fr/ lecroq/string/.

[21] M. Crochemore, A. Czumaj, L. Gasieniec, S. Jarominek, T. Lecroq, W. Plandowski, and W. Rytter, "Deux méthodes pour accélérer l'algorithme de Boyer-Moore," Théorie des Automates et Applications, Actes des 2e Journées FrancoBelges, D. Krob ed., Rouen, France, pp. 45-63, 1992.

[22] A. Apostolico and R. Giancarlo, "The Boyer-Moore-Galil string searching strategies revisited," SIAM J. Comput., vol. 15, pp. 98-105, 1986.

[23] L. Colussi, "Fastest pattern matching in strings," Journal of Algorithms, vol. 16, pp. 163-189, 1994.

[24] D. M. Sunday, "A very fast substring search algorithm," Communications of the ACM, vol. 33, pp. 132-142, 1990.

[25] R. F. Zhu and T. Takaoka, "On improving the average case of the Boyer-Moore string matching algorithm," Journal of Information Processing, vol. 10, pp. 173-177, 1987.

[26] T. Berry and S. Ravindran, "A fast string matching algorithm and experimental results," in Proceedings of the Prague Stringology Club Workshop '99, J. Holub and M. Simánek ed., Collaborative Report DC-99-05, Czech Technical University, Prague, Czech Republic, 1999, pp. 16-28.
[27] P. D. Smith, "Experiments with a very fast substring search algorithm," Software: Practice and Experience, vol. 21, pp. 1065-1074, 1991.

[28] T. Raita, "Tuning the Boyer-Moore-Horspool string searching algorithm," Software: Practice and Experience, vol. 22, pp. 879-884, 1992.

[29] G. Rozenberg and A. Salomaa, Handbook of Formal Languages: Beyond Words: Springer Verlag, 1997.

[30] R. M. Karp and M. O. Rabin, "Efficient randomized patternmatching algorithms," IBM Journal of Research and Development, vol. 31, pp. 249-260, 1987.

[31] R. Baeza-Yates and G. H. Gonnet, "A new approach to text searching," Communications of the ACM, vol. 35, p. 82, 1992.

[32] J. H. Morris and V. R. Pratt, "A linear pattern-matching algorithm," Technical Report 40, University of California, Berkeley, 1970.

[33] D. E. Knuth, J. H. Morris Jr, and V. R. Pratt, "Fast pattern matching in strings," SIAM Journal on Computing, vol. 6, p. 323, 1977.

[34] I. Simon, "String matching algorithms and automata," in R Baeza Yates, N. Ziviani (Eds.),First American Workshop on String Processing, Universidale Federal de Minas Gerais, Brazil, 1993, pp. 151-157.

[35] L. Colussi, "Correctness and efficiency of pattern matching algorithms," Information and Computation, vol. 95, pp. 225$251,1991$.

[36] Z. Galil and R. Giancarlo, "On the exact complexity of string matching: upper bounds," SIAM Journal on Computing, vol. 21, p. 407, 1992.

[37] A. Apostolico and M. Crochemore, "Optimal canonization of all substrings of a string," Information and Computation, vol. 95, pp. 76-95, 1991.

[38] C. Hancart, "Une analyse en moyenne de l'algorithme de Morris et Pratt et de ses raffinements," Actes des 2e Journées franco-belges: Théories des Automates et Applications, vol. 176, pp. 99-110.

[39] T. Lecroq, "A variation on the Boyer-Moore algorithm," Theor. Comput. Sci., vol. 92, pp. 119-144, 1992.

[40] T. Lecroq, "Experimental results on string matching algorithms," Software: Practice and Experience, vol. 25, pp. 727-765, 1995

[41] M. Crochemore and W. Rytter, Text algorithms: Oxford University Press, Inc. New York, NY, USA, 1994.

[42] G. Navarro and M. Raffinot, "A bit-parallel approach to suffix automata: Fast extended string matching," in Proceedings of the 9th Annual Symposium on Combinatorial Pattern Matching, Lecture Notes in Computer Science 1448, Springer-Verlag, Berlin,, 1998, pp. 14-33.

[43] C. Allauzen, M. Crochemore, and M. Raffinot, "Factor oracle: a new structure for pattern matching," in Proceedings of SOFSEM'99, Theory and Practice of Informatics, Lecture Notes in Computer Science 1725, J. Pavelka, G. Tel and M. Bartosek ed., Milovy,Czech Republic,Springer-Verlag, Berlin 1999, pp. 291-306.

[44] Z. Galil and J. Seiferas, "Time-space-optimal string matching* 1," Journal of Computer and System Sciences, vol. 26, pp. 280-294, 1983.

[45] M. Crochemore and D. Perrin, "Two-way string-matching," Journal of the ACM (JACM), vol. 38, pp. 650-674, 1991.

[46] C. Charras, T. Lecrog, and J. Pehoushek, "A very fast string matching algorithm for small alphabets and long patterns," in Proceedings of the 9th Annual Symposium on Combinatorial Pattern Matching , M. Farach-Colton ed.,Lecture Notes in Computer Science 1448, Piscataway, New Jersey, 1998, pp. 55-64.

[47] M. Becchi, M. Franklin, and P. Crowley, "A workload for evaluating deep packet inspection architectures," in IEEE International Symposium on Workload Characterization, 2008. IISWC 2008., 2008, pp. 79-89.

[48] Y. Fang, C. Zhifeng, D. Yanlei, T. V. Lakshman, and R. H. Katz, "Fast and memory-efficient regular expression matching for deep packet inspection," in ACM/IEEE Symposium on Architecture for Networking and Comm. systems, 2006. ANCS 2006. , 2006, pp. 93-102. 
[49] C. R. Clark and D. E. Schimmel, "Scalable pattern matching for high speed networks," in 12th Annual IEEE Symposium on Field-Programmable Custom Computing Machines, 2004. FCCM 2004, 2004, pp. 249-257.

[50] R. Sidhu and V. K. Prasanna, "Fast Regular Expression Matching Using FPGAs," in The 9th Annual IEEE Symposium on Field-Programmable Custom Computing Machines, 2001. FCCM '01., 2001, pp. 227-238.

[51] B. Michela and C. Patrick, "A hybrid finite automaton for practical deep packet inspection," in Proceedings of the 2007 ACM CoNEXT conference New York, New York: ACM, 2007.

[52] V. Dimopoulos, I. Papaefstathiou, and D. Pnevmatikatos, "A Memory-Efficient Reconfigurable Aho-Corasick FSM Implementation for Intrusion Detection Systems," in International Conference on Embedded Computer Systems: Architectures, Modeling and Simulation, 2007. IC-SAMOS 2007., 2007, pp. 186-193.

[53] K. Sailesh, D. Sarang, Y. Fang, C. Patrick, and T. Jonathan, "Algorithms to accelerate multiple regular expressions matching for deep packet inspection," in Proceedings of the 2006 conference on Applications, technologies, architectures, and protocols for computer communications Pisa, Italy: ACM, 2006.

[54] K. Sailesh, T. Jonathan, and W. John, "Advanced algorithms for fast and scalable deep packet inspection," in Proceedings of the $2006 \mathrm{ACM} / \mathrm{IEEE}$ symposium on Architecture for networking and communications systems San Jose, California, USA: ACM, 2006.

[55] L. Vespa, M. Mathew, and W. Ning, "P3FSM: Portable Predictive Pattern Matching Finite State Machine," in 20th IEEE International Conference on Application-specific Systems, Architectures and Processors, 2009. ASAP 2009., 2009, pp. 219-222.

[56] K. Sailesh, C. Balakrishnan, T. Jonathan, and V. George, "Curing regular expressions matching algorithms from insomnia, amnesia, and acalculia," in Proceedings of the 3rd ACM/IEEE Symposium on Architecture for networking and communications systems Orlando, Florida, USA: ACM, 2007, pp. $155-164$.

[57] S. Randy, E. Cristian, J. Somesh, and K. Shijin, "Deflating the big bang: fast and scalable deep packet inspection with extended finite automata," in Proceedings of the ACM SIGCOMM 2008 conference on Data communication Seattle, WA, USA: ACM, 2008.

[58] B. Raahemi, A. Kouznetsov, A. Hayajneh, and P. Rabinovitch, "Classification of Peer-to-Peer traffic using incremental neural networks (Fuzzy ARTMAP)," in Canadian Conference on Electrical and Computer Engineering, 2008. CCECE 2008., 2008, pp. 000719-000724. 